\title{
Nearest neighbor embedding with different time delays
}

\author{
Sara P. Garcia* \\ Biomathematics Group, Instituto de Tecnologia Química e Biológica, \\ Universidade Nova de Lisboa, Rua da Quinta Grande 6, 2780-156 Oeiras, Portugal \\ Jonas S. Almeida \\ Department of Biostatistics, Bioinformatics and Epidemiology, \\ Medical University of South Carolina, 135 Cannon Street, Charleston, SC 29425, USA
}

(Dated: June 23, 2018)

\begin{abstract}
A nearest neighbor based selection of time delays for phase space reconstruction is proposed and compared to the standard use of time delayed mutual information. The possibility of using different time delays for consecutive dimensions is considered. A case study of numerically generated solutions of the Lorenz system is used for illustration. The effect of contamination with various levels of additive Gaussian white noise is discussed.
\end{abstract}

(C)2005 The American Physical Society, http://link.aps.org/abstract/PRE/v71/e037204)

PACS numbers: 05.45.Tp, 05.40.Ca

Reconstructing the phase space of a dynamical system from a time series is a well-known mathematical result central to almost all nonlinear time series analysis methods (see [3] for a general introduction). It is of paramount importance as it ensures that, under certain generic conditions, such a reconstruction is equivalent to the original phase space. This equivalence ensures that differential information is preserved and allows for both qualitative and quantitative analysis. Consider a smooth deterministic dynamical system $s(t)=f\left(s\left(t_{0}\right)\right)$, either in continuous or discrete time, whose trajectories are asymptotic to a compact $d$-dimensional manifold $\mathcal{A}$. When performing $k$-dimensional measurements, where $k=1, \ldots, d$, a function $\mathbf{x}_{(i)}=h[s(t=i \times \delta)]$ relates the states of the dynamical system throughout time and a time series of measured points, where $\mathbf{x}_{(i)} \in \mathbb{R}^{k}, i=1, \ldots, n ; n$ is the total number of sampled points, and $\delta$ is the sampling time. As a consequence of our ignorance on the system, or of limitations of the measurement apparatus, or simply because it is too costly, $d$-dimensional measurements are typically not made [1, $[6]$. In this report we will only address scalar measurements, that is, $k=1$. Phase space reconstruction by time delay embedding is a method of generating an $m$-dimensional manifold that is equivalent to the original $d$-dimensional manifold, by means of a matrix of delaycoordinate vectors. Consider a column vector time series $\mathbf{x}_{(i)}$. Define an $m$-dimensional matrix of delay-coordinate column vectors by adding together displaced copies of the time series, $\mathbf{X}=\left[\mathbf{x}_{(i)}, \mathbf{x}_{(i+\tau)}, \ldots, \mathbf{x}_{(i+(m-1) \tau)}\right]$. Such matrix $\mathbf{X}_{[n-(m-1) \times \tau, m]}$ is called an embedding matrix, and two parameters need to be optimally estimated. The first is the time delay $\tau$, which quantifies the time displacement between successive delay-coordinate vectors. The

*spinto@itqb.unl.pt second is the embedding dimension $m$, which quantifies the number of such delay-coordinate vectors. In this report we only address the estimation of $\tau$, by suggesting a nearest neighbor based procedure and comparing it to the standard use of time delayed mutual information. Though in the limit of infinite data and infinite precision $\tau$ may be set to any arbitrary value, a balance between relevance and redundancy [1] must be accomplished for real data. When $\tau$ is too small, the elements of the delay-coordinate vectors will mostly be around the bisectrix of the phase space and, consequently, the reconstruction will not be satisfactory. On the contrary, if $\tau$ is too large the delay-coordinate vectors will become increasingly uncorrelated, with the consequent loss of ability to recover the underlying attractor. In addition, using a time delay larger than necessary will render fewer data points for the reconstruction. This may be particularly limiting for the study of biological systems, where data sets are often not long. The most common procedure for selecting $\tau$ is using the first minimum of time delayed mutual information, as proposed by Fraser and Swinney [2]: $I\left(x_{(i)}, x_{(i+\tau)}, \tau\right)=H\left(x_{(i)}\right)+H\left(x_{(i+\tau)}\right)-H\left(x_{(i)}, x_{(i+\tau)}\right)=$ $\sum p\left(x_{(i)}, x_{(i+\tau)}\right) \log _{2} \frac{p\left(x_{(i)}, x_{(i+\tau)}\right)}{p\left(x_{(i)}\right) p\left(x_{(i+\tau)}\right)}$, where $H(x)$ is the Shannon entropy [8]. Nonetheless its widespread use, some drawbacks can be pointed out to this selection criterion. The first is that probabilities are estimated by creating a histogram for the probability distribution of the data, which depends on a heuristic choice of number of bins, for example, $\log _{2}$ of the total number of points [9]. Therefore, $I$ depends on the partitioning. The second drawback is that it contains no dynamical information, which might be incorporated by considering transition rather than static probabilities, but such correction is usually not made [7]. The third is that the selection criterion presented by Fraser and Swinney [2], though generalized to higher dimensions, was established for two-dimensional embeddings [1], and does not neces- 
sarily hold for higher dimensional embeddings, as shown below. Finally, a fourth drawback [1] is associated with the fact that, when the purpose is solely to maximize statistical independence [2], there is no obvious reason to choose the first minimum over others. We propose an alternative measure for selecting time delays, based on nearest neighbor estimations. This nearest neighbor measure is inspired by the false nearest neighbors algorithm proposed by Kennel et al. [4]. With minimal assumptions, this measure is based solely on topological and dynamical arguments documented by the data. We do not address the estimation of $m$. The embedding theorem proposed by Takens [10] guarantees a solution. It states that if a map from the original $d$-dimensional phase space $\mathcal{A}$, to the reconstructed $m$-dimensional phase space is generic, when $m \geq(2 d+1)$ that map is a diffeomorphism on $\mathcal{A}$, that is, an embedding, or a smooth one-toone map with a smooth inverse. This one-to-one property implies that if the system is deterministic, distinct points on the attractor $\mathcal{A}$ are mapped to distinct points under the embedding map [6]. Nevertheless, Takens result is only a sufficient condition according to Kennel et al. [4], who propose the use of false nearest neighbors $(\mathrm{F})$ as a criteria. Their algorithm considers the ratio of Euclidean distances between a point and its nearest neighbor, first on a $m$-dimensional and then on a $(m+1)$-dimensional space. If the ratio is greater than a given threshold, these points are referred to as $\mathrm{F}$, that is, points that appear to be nearest neighbors not because of the dynamics, but because the attractor is being viewed in an embedding space too small to unfold it. The procedure is repeated for all points in the time series. As the fraction of $F$ as a function of the embedding space dimension decreases for deterministic systems, when its value is zero, the underlying attractor is unfolded and $m$ can be optimally estimated.

Considering the problem of optimally selecting time delays, we will compare two different approaches. The first is a standard procedure and uses the first minimum of the time delayed mutual information to set $\tau$ for all dimensions [2]. The second, the one we propose, uses the first minimum of a nearest neighbor measure to set the time delay for each dimension. Therefore, this second procedure is iterative and introduces two novelties: using a nearest neighbor based measure instead of the time delayed mutual information, and using different time delays for consecutive dimensions, as the standard use of the same $\tau$ value is an assumption out of convenience and not imposed by any theoretical argument [1]. In both cases, the embedding dimension is estimated as the fraction of $\mathrm{F}$ decreases to zero. The implementation of the standard procedure is described below. (i) Consider an initial column vector time series $\mathbf{x}_{(i)}$. For each $\tau$ being tested, $\tau=1, \ldots, \frac{1}{10} n$, build a temporary embedding matrix $\mathbf{T}=\left[\mathbf{x}_{(i)}, \mathbf{x}_{(i+\tau)}\right]$ out of two column vectors $\mathbf{x}_{(i)}$ and $\mathbf{x}_{(i+\tau)}$. The upper limit for $\tau$ is set arbitrarily. (ii) Estimate the time delayed mutual information $I\left(\mathbf{x}_{(i)}, \mathbf{x}_{(i+\tau)}\right)$. (iii) Select the first minimum from the profile of $I$ vs $\tau$, which will be the optimal time delay for all dimensions (columns) of the final embedding matrix $\mathbf{X}$. (iv) Estimate the percentage of $\mathrm{F}$ (algorithm in [4]) as a function of the dimensionality of the embedding matrix. The optimal embedding dimension is set when the fraction of $\mathrm{F}$ drops to 0 . As $\tau$ is the same for all dimensions it will be referred to as a fixed time delay, and the final embedding matrix will be $\mathbf{X}=\left[\mathbf{x}_{(i)}, \mathbf{x}_{(i+\tau)}, \ldots, \mathbf{x}_{(i+(m-1) \tau)}\right]$. The implementation of the proposed algorithm is as follows. (i) Consider an initial column vector time series $\mathbf{x}_{(i)}$. For each $\tau$ being tested, $\tau=1, \ldots, \frac{1}{10} n$, build a temporary embedding matrix $\mathbf{T}=\left[\mathbf{x}_{(i)}, \mathbf{x}_{(i+\tau)}\right]$. (ii) For each twodimensional point, that is, for each row in matrix $\mathbf{T}$, estimate its (two-dimensional) nearest neighbor. Calculate the Euclidean distance between them, $d_{E 1}$. (iii) Consider both points one sampling unit ahead and calculate the new Euclidean distance between them, $d_{E 2}$. (iv) Estimate $d_{E 2} / d_{E 1}$ and save the number of distance ratios larger than 10. That fraction will be referred to as $N$. The threshold value, though heuristically set, is justified by numerical studies [4] and has low parametric sensitivity. (v) Select the first minimum from a profile of $N$ vs $\tau$, which will be the optimal time delay for this first embedding cycle, $\tau_{1}$. We define an embedding cycle as each iteration [steps (i) to (vi)] that adds another dimension to the embedding matrix. (vi) Estimate the percentage of F. Save that value as a function of the dimensionality of the temporary embedding matrix $\mathbf{T}$. (vii) Consider now matrix $\mathbf{X}=\left[\mathbf{x}_{(i)}, \mathbf{x}_{\left(i+\tau_{1}\right)}\right]$ as the starting point for the second embedding cycle. For each $\tau$ being tested, build a temporary embedding matrix $\mathbf{T}=\left[\mathbf{x}_{(i)}, \mathbf{x}_{\left(i+\tau_{1}\right)}, \mathbf{x}_{(i+\tau)}\right]$. (viii) Repeat steps (ii) to (vii), considering that points are now three- and more dimensional, until the fraction of $\mathrm{F}$ drops to 0 . As there will be a vector of $\tau$ values $\left[\tau_{1}, \ldots, \tau_{(m-1)}\right]$, this procedure is said to use different time delays, and the final embedding matrix will be $\mathbf{X}=\left[\mathbf{x}_{(i)}, \mathbf{x}_{\left(i+\tau_{1}\right)}, \mathbf{x}_{\left(i+\tau_{2}\right)}, \ldots, \mathbf{x}_{\left(i+\tau_{(m-1)}\right)}\right]$.

The Lorenz system [5] of differential equations $\dot{x}=$ $\sigma(y-x), \dot{y}=x(\rho-z)-y, \dot{z}=x y-\beta z$, with parameters $\sigma=10, \rho=28, \beta=8 / 3$ will be used as a case study. The equations were numerically integrated with a $4-5$ th order Runge-Kutta algorithm and sampled at $\delta=0.01$ intervals. Transients were removed. We will consider a first data set, referred to as $L(X)$, which is the noise-free $x$-coordinate of Lorenz system. We will also consider a second data set, referred to as $L\left(X_{\eta}\right)$, consisting of the noise-free $x$-coordinate of the Lorenz system contaminated with additive Gaussian white noise of mean zero and variance $0.05,1,2,3$, or 5 . For the major part of this report, noise of variance 1 will be used. The other variances will be used later to further document the effect of noise on the $\tau$ selecting procedures. Real systems may also be contaminated with dynamical noise, though we do not address such possibility here. Each data set includes a total of 8000 points, $\left(\frac{1}{8}\right)$ th of which is plotted in Fig. 1.

The profiles for selecting $\tau$ are displayed in Fig. 2 for the first embedding cycle, and in Fig. 3 for the second 

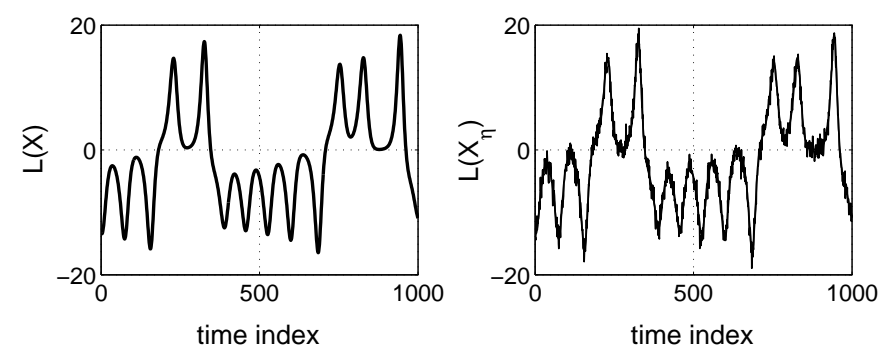

FIG. 1: Data sets: noise-free $x$-coordinate of the Lorenz system $[L(X)]$, and contaminated with additive Gaussian white noise of mean 0 and variance $1\left[L\left(X_{\eta}\right)\right]$.

embedding cycle. Dashed lines represent $I$ profiles, while solid lines represent $N$ profiles. Because the standard $\tau$ selecting procedure uses the same time delay for all dimensions, only $N$ profiles are represented in Fig. 3. Displayed on the upper panels are the profiles for $L(X)$ [Fig. 2(a) and Fig. 3(a)] and $L\left(X_{\eta}\right)$ of variance 1 [Fig. 2(b) and Fig. 3(b)]. On the lower panel, zoomed out versions of those same profiles are plotted to document behavior beyond dynamic coupling. An arrow indicates the global minimum of $N$ for the noise-free scenario [Fig. 2(a) and (c), Fig. 3(a) and (c), solid line]. That same value can still be identified for the first embedding cycle of the noisy data set $L\left(X_{\eta}\right)$, though it is no longer a global minimum [Fig. 2(d), solid line]. As explained previously, a value for $\tau$ is selected from the first minimum of the $I$ profile for both $L(X)$ and $L\left(X_{\eta}\right)$ [Fig. 2(a) and (b), dashed line]; and a value for $\tau_{1}$ is selected from the first minimum of the $N$ profile for both $L(X)$ and $L\left(X_{\eta}\right)$ [Fig. $2(\mathrm{a})$ and (b), solid line]. For the second embedding cycle, an asterisk indicates $\tau_{1}$, that is, the value selected in the first embedding cycle, while a circle indicates $\tau_{2}$, that is, the first minimum of this new $N$ profile [Fig. 3(a)].

Three main conclusions can be drawn from examining the $\tau$ selecting profiles from both $I$ and $N$ for the first and second embedding cycles. The first is that only $N$ retains the inverse relationship with structure disclosure, that is, unlike $I, N$ values return to higher levels when the time delay is too long for dynamical coupling to be retained [Fig. 2(c) and Fig. 3(c), arrow]. This global minimum suggests an upper limit for the efficient selection of $\tau$, beyond which statistical independence reflects dynamic decoupling, and provides the strongest argument for the use of $N$ over $I$. The effect of noise will be discussed later. The second observation is that the profiles for both embedding cycles are strikingly different, indicating that reusing the time delay from a previous embedding cycle is not an efficient procedure, as the $N$ profile peaks at $\tau_{1}$ [Fig. 3(a), asterisk]. This peaking, an interesting but presently unclear feature, was consistently observed for all embedding cycles, and not only for the data sets analyzed here but also for other systems, such as the Rössler attractor 11], not shown here for space constraints. The third conclusion refers to the disruptive effect of additive noise, particularly clear in Fig. 3(b). To further docu-
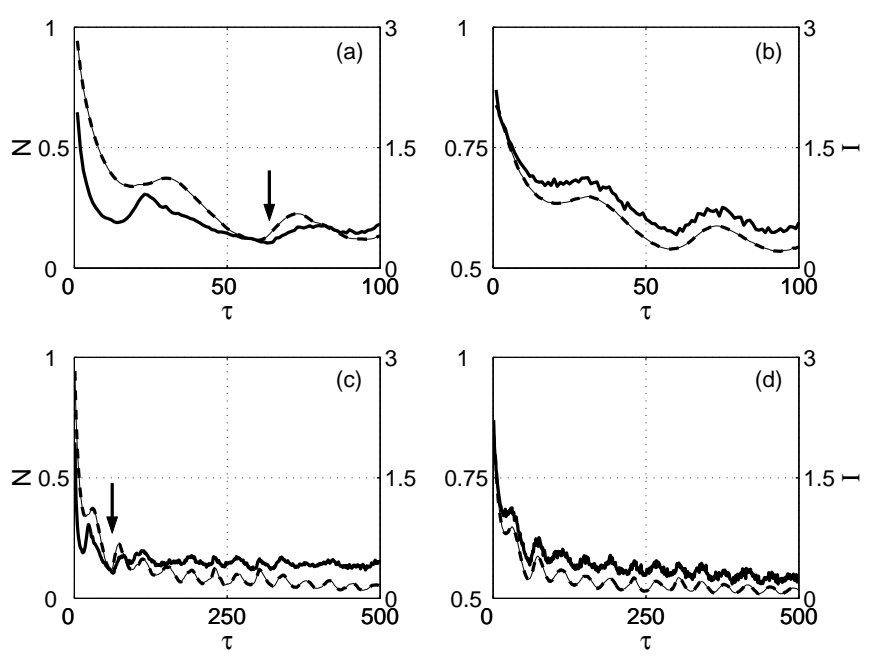

FIG. 2: First embedding cycle profiles for $\tau$ selection from $I$ (dashed lines) and $N$ (solid lines). Upper panel: (a) $L(X)$ and (b) $L\left(X_{\eta}\right)$ of variance 1 . Lower panel: zoomed out (c) $L(X)$ and $(\mathrm{d}) L\left(X_{\eta}\right)$ of variance 1 . An arrow indicates the global minimum of $N$ for the noise-free scenario [(a) and (c), solid line].
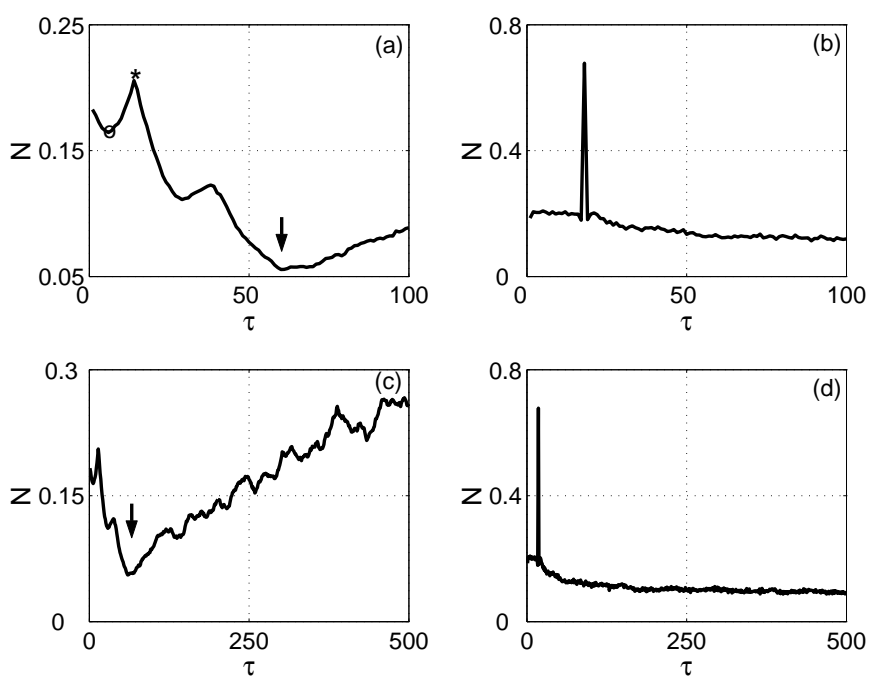

FIG. 3: Second embedding cycle profiles for $\tau$ selection from $N$. Upper panel: (a) $L(X)$ and (b) $L\left(X_{\eta}\right)$ of variance 1 . Lower panel: zoomed out (c) $L(X)$ and (d) $L\left(X_{\eta}\right)$ of variance 1. An arrow indicates the global minimum of $N$ for the noisefree scenario $[(\mathrm{a})$ and $(\mathrm{c})]$. An asterisk indicates $\tau_{1}$, while a circle indicates $\tau_{2}[(\mathrm{a})]$.

ment such effect, profiles from $N$ for the second embedding cycle and additive Gaussian white noise of different variances are displayed in Fig. 4. The noise-free scenario [Fig. 4(a), as in Fig. 3(a)] is compared to additive Gaussian white noise of mean 0 and variance 0.05 [Fig. 4(b)], 1 [Fig. 4(c), as in Fig. 3(b)], 2 [Fig. 4(d)], 3 [Fig. 4(e)], and 5 [Fig. $4(\mathrm{f})]$. All profiles peak exactly at $\tau_{1}$, as had been previously observed in Fig. 3. Interestingly, the $\tau$ value that is a global minimum in the noise-free scenario 
[Fig. 4(a), arrow] is in most cases still identifiable. This feature may be a helpful guideline, for the global minimum, though being a suboptimal choice, sets the upper limit for the selection of $\tau$ values.
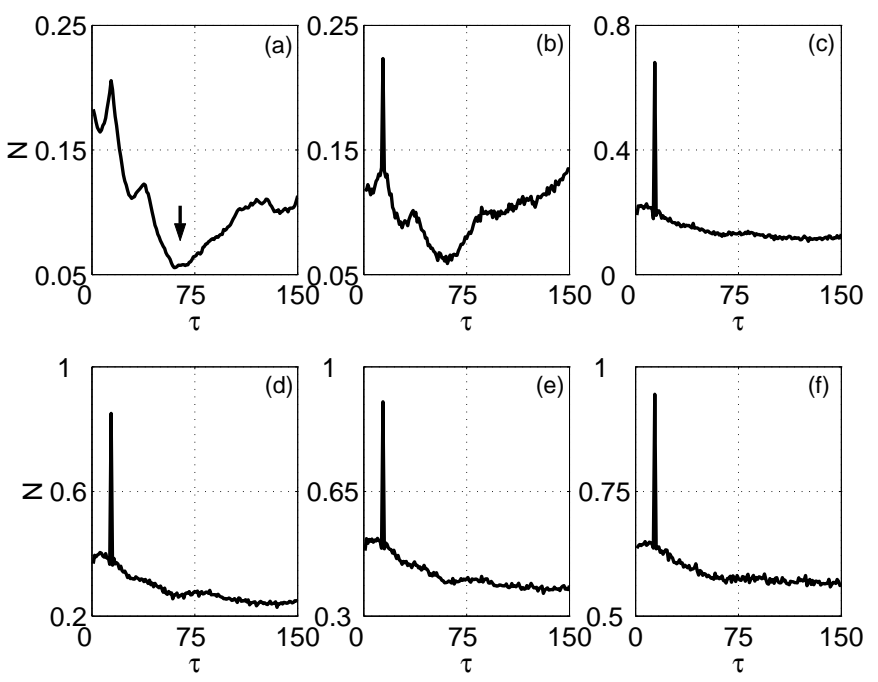

FIG. 4: Second embedding cycle profiles for $\tau$ selection from $N$ : (a) $L(X)$ [as in Fig. 3(a)] and $L\left(X_{\eta}\right)$ contaminated with additive Gaussian white noise of mean 0 and variance (b) 0.05, (c) 1 [as in Fig. 3(b)], (d) 2, (e) 3, and (f) 5. An arrow indicates the global minimum of $N$ for the noise-free scenario [as in Fig. 3(a)].

The second part of phase space reconstruction implies the estimation of the embedding dimension. Fig. 5 documents the profiles of $\mathrm{F}$ for increasing $m$ values, for the noise-free $L(X)$ data set. A dashed line represents the conventional use of $I$ and fixed time delays, while a thick solid line represents using $N$ and selecting different time delays. The reconstruction using the later is more efficient, in the sense that, though both $I$ and $N$ suggest $m=3$ as the optimal embedding dimension, the percentage of $\mathrm{F}$ when $m=2$ is lower for $N$. We have argued that the global minimum of $N$ from the noise-free scenario would be an upper limit for the efficient selection of $\tau$ values. A thin solid line represents selecting the global minimum of $N$ as the $\tau$ value for all embedding cycles, and it is clearly a suboptimal choice. This further confirms the relevance of the global minimum as a criterion for upper-limiting the region where the selection of time delays should be made.

In summary, the nearest neighbor measure we propose, unlike mutual information, retains the inverse relationship with structure disclosure. This is an extremely useful feature for analyzing noisy time series as it allows for the determination of an upper limit to an efficient selection of time delays. Another extremely important result is that the use of different time delays is more efficient than the conventional use of a fixed time delay.

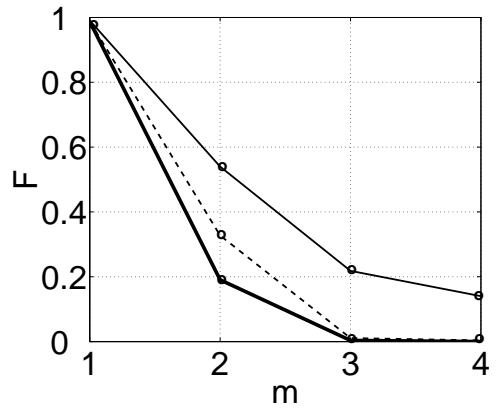

FIG. 5: Profiles for $m$ selection from $\mathrm{F}$ for $L(X)$ : selecting a fixed $\tau$ from the first minimum of $I$ (dashed line), different $\tau$ values from the first minimum of $N$ (thick solid line), and a fixed $\tau$ from the global minimum of $N$ (thin solid line).

\section{Acknowledgments}

The authors thank S. Vinga and the referees for insightful suggestions. This work was supported by grants SFRH/BD/1165/2000 and POCTI/1999/BSE/34794 from Fundação para a Ciência e a Tecnologia, Portugal, and by the National Heart, Lung and Blood Institute (NIH) Proteomics Initiative through contract N01-HV28181 (D Knapp, PI).
[1] M. Casdagli, S. Eubank, J.D. Farmer, and J. Gibson. Physica (Amsterdam), 51D:52-98, 1991.

[2] A.M. Fraser and H.L. Swinney. Phys. Rev. A, 33:11341140, 1986.

[3] H. Kantz and T. Schreiber. Nonlinear Time Series Analysis. Cambridge University Press, Cambridge, UK, 1997.

[4] M.B. Kennel, R. Brown, and H.D.I. Abarbanel. Phys. Rev. A, 45:3403-3411, 1992.

[5] E. Lorenz. J. Atmos. Sci., 20:130-141, 1963.

[6] T. Sauer, J.A. Yorke, and M. Casdagli. J. Stat. Phys., 65:579-616, 1991.
[7] T. Schreiber. Phys. Rev. Lett., 85:461-464, 2000.

[8] C.E. Shannon and W. Weaver. The Mathematical Theory of Communication. University of Illinois Press, Urbana, IL, 1967.

[9] H.A. Sturges. J. Amer. Statist. Assoc., 21:65-66, 1926.

[10] F. Takens. Dynamical Systems and Turbulence, volume 898 of Lecture Notes in Mathematics, pages 366-381. Springer-Verlag, Berlin, 1981.

[11] O. Rössler. Z. Naturforsch., 31:1168-1172, 1976. 